\title{
Clinical impact of extending after-hours radiology coverage for emergency department computed tomography imaging
}

This article was published in the following Dove Press journal:

Open Access Emergency Medicine

3 May 2014

Number of times this article has been viewed

\author{
Samer Dabbo' \\ Catherine Varner ${ }^{2,3}$ \\ Robert Bleakney ${ }^{1,2}$ \\ Howard Ovens 2,3 $^{2}$ \\ 'University Health Network, \\ Department of Medical Imaging, \\ University of Toronto, ${ }^{2}$ Division of \\ Emergency Medicine, Schwartz/ \\ Reisman Emergency Medicine \\ Institute, Mount Sinai Hospital, \\ ${ }^{3}$ Department of Family and \\ Community Medicine, University of \\ Toronto, Toronto, Canada
}

Background: Academic emergency departments (EDs) are often reliant on preliminary interpretation by radiology residents for after-hours computed tomography (CT) images. Identifying residents' errors in diagnostic interpretation and ensuring appropriate contact with affected patients are areas of continuing concern.

Objective: The Mount Sinai Hospital ED and Medical Imaging Department in Toronto, Canada sought to examine the clinical impact of extending reporting hours of senior attending radiologists for ED patients undergoing CT imaging.

Methods: All evening CT studies were read by the on-call sub-specialist staff radiologist before $10 \mathrm{pm}$; while studies done after $10 \mathrm{pm}$ were read by $8 \mathrm{am}$, permitting review of final reports by the ordering ED physician. A retrospective review of radiology and ED metrics was performed on ED patients undergoing CT imaging 12 weeks before and 12 weeks after implementation of the extended reading hours.

Results: In the 12 weeks prior to implementation of extended senior attending radiologist coverage, $871 \mathrm{CT}$ scans were performed as compared to $944 \mathrm{CT}$ scans after implementation. Time from performance of CT scan to obtaining a dictated report decreased from 10.4 hours to 2.8 hours $(P<0.001)$, and time from performance of CT scan to report verification by the radiologist decreased from 29.7 hours to 9.4 hours $(P<0.001)$. There were no statistically significant changes in ED length of stay, rates of admission, or rates of consultation. However, there was a significant reduction in (median) time taken for ED physicians to resolve discrepant reports in the radiology information system queue (20.7 hours versus 13.3 hours, $P<0.001$ ).

Conclusion: The extension of reporting hours reduced the time for ED physicians to review discrepant reports, while balancing educational needs of residents. This project has been considered a success by stakeholders and has now been implemented on a permanent basis.

Keywords: quality assurance, clinical competence, diagnostic errors, interns, residents

\section{Introduction}

Academic emergency departments (EDs) are often reliant on preliminary interpretation by radiology residents for after-hours computed tomography (CT) images. Radiology residents provide preliminary interpretations which then receive final interpretation by a senior attending radiologist, usually the following day. Preliminary interpretations allow for timely treatment and referral of ED patients, although identifying residents' errors in diagnostic interpretation and ensuring appropriate contact with affected patients are areas of continuing concern. ${ }^{1-3}$

The incidence of major diagnostic errors may be considered low; however, between $1 \%-8 \%$ of errors occur in radiology residents' preliminary interpretations, and delay in recognizing diagnostic errors can result in adverse clinical outcomes. ${ }^{4-8}$ Therefore, our
Correspondence: Samer Dabbo

377I Althorpe Circle, Mississauga, ON, L5N7G4, Canada

Email s.dabbo@utoronto.ca 
ED and medical imaging department sought to examine the clinical impact of extending reporting hours of senior attending radiologists for ED patients undergoing $\mathrm{CT}$ imaging.

\section{Methods}

The Mount Sinai urban teaching hospital in the city of Toronto, Canada extended after-hours coverage for senior attending radiologists on August 1, 2011. Prior to implementation of this extended coverage, senior attending radiologists provided final interpretation of CT imaging from 8 am to $5 \mathrm{pm}$. After implementation of the extended hours, senior attending radiologists interpreted studies in two batches. All evening studies were read by the on-call sub-specialist staff radiologist before $10 \mathrm{pm}$, while all studies done after $10 \mathrm{pm}$ were read before 8 am, permitting review of final reports by the ordering ED physician while balancing lifestyle issues for radiologists on call and preserving opportunities for independent practice by radiology residents. Approval from the Mount Sinai Hospital Research Ethics Board was granted prior to initiating this study.

We performed a retrospective review of all ED patients undergoing CT imaging 12 weeks before and 12 weeks after implementation. Radiology metrics, such as time elapsed from when the CT scan was ordered to when it was performed, and time elapsed from when the scan was performed to when it was verified by the reporting physician, were collected from the radiology information systems (RIS) computerized database. ED metrics for patients undergoing CT imaging, such as length of stay, were collected from the computerized ED patient information management system.

All time metrics and rates were compared with $P$-values calculated using a Mann-Whitney test and analyzed using SAS statistical software version 9.3 (SAS Institute Inc., Cary, NC, USA).

\section{Results}

In the 12 weeks prior to implementation of extended senior attending radiologist coverage, $871 \mathrm{CT}$ scans were performed as compared to $944 \mathrm{CT}$ scans after implementation. Time elapsed from ordering the CT to when the CT was performed was almost unchanged (1.6 hours versus [vs] 1.5 hours). Time from performing a CT to having a dictated report decreased from 10.4 hours to 2.8 hours $(P<0.001)$, and time from performing the scan to report verification by the radiologist decreased from 29.7 hours to 9.4 hours $(P<0.001)$. There was also a reduction in the number of cases receiving a preliminary report $(89.6 \%$ vs $70.2 \%, P<0.001)$.

There were no statistically significant changes in the length of stay in the ED ( 7.1 hours vs 7.8 hours), rates of admission (25.7\% vs $28.0 \%$ ), or rates of consultation (37.3\% vs $37.9 \%)$. However, there was a significant reduction in (median) time taken for ED physicians to resolve discrepant reports in the RIS queue (20.7 hours vs 13.3 hours, $P<0.001)$. Results are summarized in Tables 1 and 2 .

\section{Discussion}

A senior attending radiologist's interpretation of CT imagery is preferable for ensuring ED patients' safety and quality of care, though attending-level staffing is not feasible in most academic institutions 24 hours a day. However, by extending senior attending radiologists' hours in the manner described, final reports were verified 20 hours sooner than prior to this initiative's implementation. This time reduction allowed for a decreased time to reconcile and remove discrepant reports from the RIS queue. This outcome is clinically important, because it represents patients being contacted, if needed, approximately 7 hours sooner for discrepant reports.

Additional ED metrics, such as length of stay, were not affected by this change in practice. These outcomes imply that ED physicians utilize the first available report, whether preliminary or final, to refer patients, and CT imaging is not the only factor affecting these outcomes.

Although there was a significant decrease in the percentage of after-hours cases receiving a preliminary resident's report, residents still entered reports on a large number of studies $(>70 \%)$. The new model has not affected the graded call system that is in place to integrate junior residents. Staff and fellows are still available overnight if a resident

Table I Radiology metrics before and after extended radiology coverage implemented

\begin{tabular}{llll}
\hline $\begin{array}{l}\text { Radiology metrics } \\
\text { (time elapsed/\%) }\end{array}$ & $\begin{array}{l}\text { Before extended } \\
\text { radiology coverage }\end{array}$ & $\begin{array}{l}\text { After extended } \\
\text { radiology coverage }\end{array}$ & $\begin{array}{l}P \text {-value } \\
\text { Ordered to performed (hours) }\end{array}$ \\
Performed to preliminary report (hours) & 1.6 hours & 1.5 hours & 0.889 \\
Performed to dictated (hours) & 1.1 hours & 0.9 hours & $0.05 \mathrm{I}$ \\
Performed to verified (hours) & 10.4 hours & 2.8 hours & $<0.00 I^{*}$ \\
Cases with a preliminary report (\%) & 29.7 hours & 9.4 hours & $<0.00 I^{*}$ \\
\hline
\end{tabular}

Note: *Denotes statistical significance. 
Table 2 ED metrics before and after extended radiology coverage implemented

\begin{tabular}{|c|c|c|c|}
\hline Emergency department metrics & $\begin{array}{l}\text { Before extended } \\
\text { radiology coverage }\end{array}$ & $\begin{array}{l}\text { After extended } \\
\text { radiology coverage }\end{array}$ & $P$-value \\
\hline Percentage of patients getting CT scan & $9.0 \%$ & $8.4 \%$ & 0.604 \\
\hline Median length of stay for all patients in ED (hours) & 3.2 hours & 3.3 hours & 0.083 \\
\hline Median length of stay for patients getting CT scan (hours) & 7.I hours & 7.8 hours & 0.674 \\
\hline Rate of consults for patients with CT scans & $37.3 \%$ & $37.9 \%$ & 0.751 \\
\hline Rate of admission for patients with CT scans & $25.7 \%$ & $28.0 \%$ & 0.187 \\
\hline Time to resolve from queue (hours) & 20.7 hours & I 3.3 hours & $<0.001 *$ \\
\hline
\end{tabular}

Note: *Denotes statistical significance.

Abbreviations: $\mathrm{CT}$, computed tomography; ED, emergency department.

has a question regarding appropriateness, protocol, and interpretation.

The additional cost of the new after-hours reporting model was minimal. Since all the cases were previously being dictated (albeit with longer turn-around time), there was no extra income from reporting the cases sooner. The after-hours premiums/bonuses paid to staff are minimal, and impacts of reimbursement costs are only small.

\section{Limitations}

Unfortunately, this study was unable to quantify the effect on all ED quality improvement metrics such as return visits to the ED, ED call-backs, and patient morbidity from errors in preliminary imaging reports. Given that previous studies indicate major errors in residents' preliminary reports are rare, a future study analyzing such outcomes would need to be larger and involve multiple hospital sites. ${ }^{4-8}$ Additionally, this study is only generalizable to hospitals where ED physicians and radiology residents provide preliminary, after-hours reports, and radiologic and ED processes may differ from hospitals with different geographic and patient demographics.

\section{Conclusion}

In conclusion, the intervention described reduced the time for ED physicians to review discrepant reports, while balancing educational needs of residents and lifestyle concerns of staff. It has been considered a success by stakeholders (emergency department, radiology staff and radiology residents) and has now been implemented on a permanent basis.

\section{Disclosure}

The authors report no conflicts of interest in this work.

\section{References}

1. [No authors listed]. Radiology discrepancies plaguing ED managers. ED Manag. 2005;17(7):76-78.

2. Strub WM, Vagal AA, Tomsick T, Moulton JS. Overnight resident preliminary interpretations on CT examinations: should the process continue? Emerg Radiol. 2006;13(1):19-23.

3. Lal NR, Murray UM, Eldevik OP, Desmond JS. Clinical consequences of misinterpretations of neuroradiologic CT scans by on-call radiology residents. AJNR Am J Neuroradiol. 2000;21(1):124-129.

4. Carney E, Kempf J, DeCarvalho E, Yudd A, Nosher J. Preliminary interpretations of after-hours $\mathrm{CT}$ and sonography by radiology residents versus final interpretations by body imaging radiologists at a level I trauma center. AJR Am J Roentgenol. 2003;181(2):367-373.

5. Stevens KJ, Griffiths KL, Rosenberg J, Mahadevan S, Zatz LM, Leung AN. Discordance rates between preliminary and final radiology reports on cross-sectional imaging studies at a level 1 trauma center. Acad Radiol. 2008;15(10):1217-1226.

6. Walls J, Hunter N, Brasher PM, Ho SG. The DePICTORS Study: discrepancies in preliminary interpretation of CT scans between on-call residents and staff. Emerg Radiol. 2009;16(4):303-308.

7. Chung JH, Strigel RM, Chew AR, Albrecht E, Gunn ML. Overnight resident interpretation of torso $\mathrm{CT}$ at a level 1 trauma center an analysis and review of the literature. Acad Radiol. 2009;16(9):1155-1160.

8. Ruutlainen AT, Scanlon MH, Itri JN. Identifying benchmarks for discrepancy rates in preliminary interpretations provided by radiology trainees at an academic institution. $J$ Am Coll Radiol. 2011;8(9): 644-648.
Open Access Emergency Medicine

\section{Publish your work in this journal}

Open Access Emergency Medicine is an international, peer-reviewed, open access journal publishing original research, reports, editorials, reviews and commentaries on all aspects of emergency medicine. The manuscript management system is completely online and includes a very quick and fair peer-review system, which is all easy to use.

\section{Dovepress}

Visit http://www.dovepress.com/testimonials.php to read real quotes from published authors. 